\title{
Scar pregnancy: a rare complication of caesarean section
}

\author{
A. Maria Emilia Abadilla • David Jaspan • \\ Vani Dandolu
}

Received: 9 October 2007 / Accepted: 21 December 2007 /Published online: 30 January 2008

(C) Springer-Verlag 2008

\begin{abstract}
In this paper, we report a rare type of ectopic pregnancy implanted in a previous caesarean scar. Scar pregnancy was diagnosed at six weeks gestation in a woman with three prior caesarean deliveries. Management with the usual dose of Methotrexate was unsuccessful. The patient continued to have pelvic pain, the beta human chorionic gonadotropin ( $(\mathrm{hCG}$ ) increased and there was persistent foetal cardiac activity. Subsequently, dilatation and curettage was performed under ultrasound guidance without complications. We conclude that scar pregnancy presents a diagnostic and therapeutic challenge to the clinician and the optimal management strategy needs to be explored.
\end{abstract}

Keywords Scar pregnancy - Ectopic pregnancy · Caesarean complications · Caesarean scar pregnancy

\section{Introduction}

Scar pregnancy is a rare form of ectopic pregnancy and is described as the implantation of pregnancy in a previous caesarean section scar. Because of its rarity, diagnosis is challenging and its treatment has not been standardised in the face of potentially catastrophic complications.

We report a case of caesarean scar pregnancy which was diagnosed early with transvaginal sonography and treated medically and surgically as discussed below.

A. M. E. Abadilla $\cdot$ D. Jaspan

Albert Einstein Medical Center,

Philadelphia, PA, USA

V. Dandolu $(\bowtie)$

Temple University Hospital,

3401 N Broad street,

Philadelphia, PA 19140, USA

e-mail: vani.dandolu@temple.edu
Case

A 26-year-old G7P2406 presented to the Albert Einstein Medical Center emergency department with complaints of mild cramping lower abdominal pain and 5.5 weeks of amenorrhoea. Her obstetric history was significant for two low transverse caesarean deliveries and a recent repeat caesarean section 5 months previously, performed via a classical incision secondary to dense adhesions and complicated further by bowel resection. On examination, the patient was stable, had no vaginal bleeding, her cervix was closed, uterus anteverted, normal-sized and mildly tender, with no adnexal masses. Transvaginal ultrasound revealed a gestational sac measuring $8.0 \times 5.3 \times 5.9 \mathrm{~cm}$, corresponding to a gestational age of 5 weeks and 2 days, positioned approximately at the level of the caesarean section scar with the myometrium over the sac slightly thinned compared to the posterior myometrium.

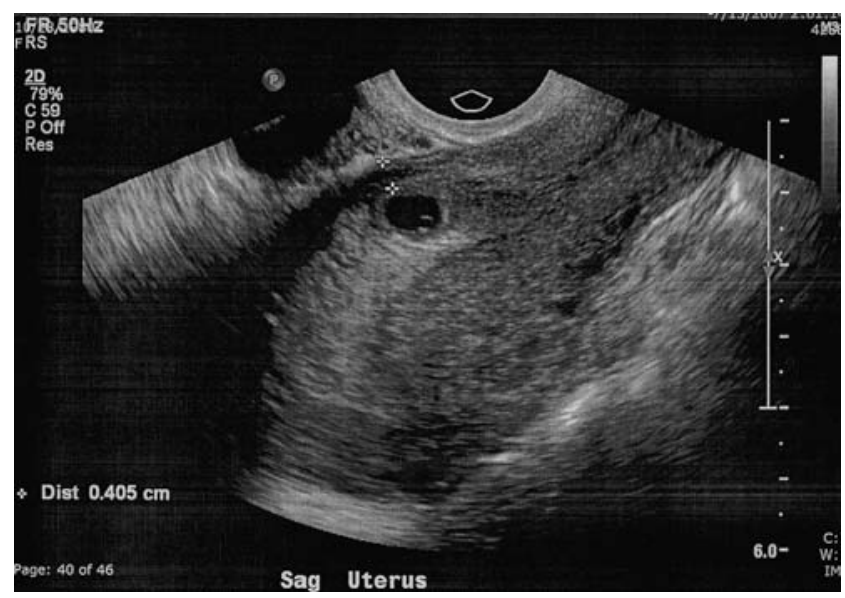


Expectant management was discussed in detail and the patient was to return in a week for a confirmatory repeat transvaginal ultrasound. One week later, vaginal sonographic examination showed that the gestational sac was still eccentrically located in the lower anterior aspect of the uterus adjacent to the scar, within which was a yolk sac and a live embryo at 6 weeks gestation.

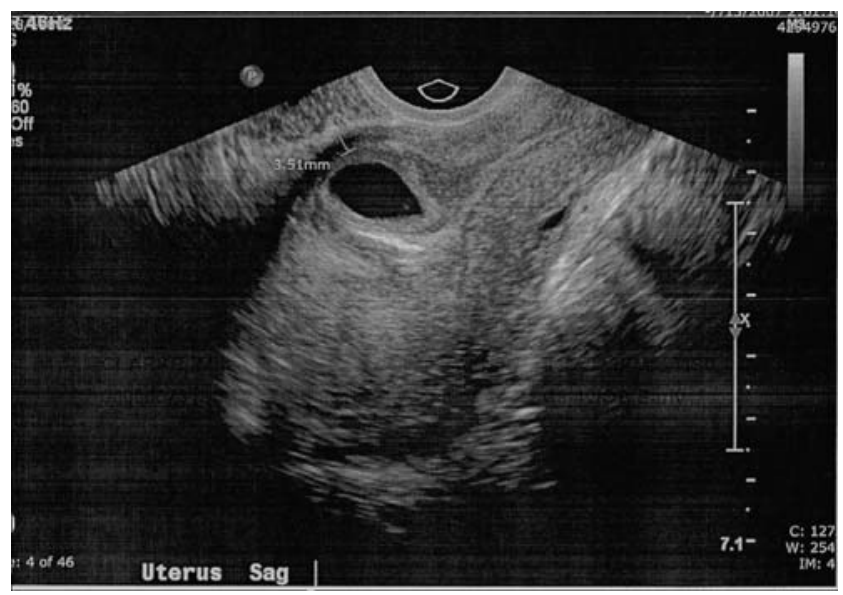

The myometrium overlying the anterior portion of the gestational sac has become even thinner from the previous study.

Baseline laboratory exams were Rh-positive, haemoglobin of $10.3 \mathrm{~g} / \mathrm{dL}$ and beta human chorionic gonadotropin (BhCG) of $14,100 \mathrm{mIU} / \mathrm{mL}$. Important issues considered were the patient's stable status and her history of multiple abdominal surgeries. The patient was consented and received one dose of $89 \mathrm{mg}$ of Methotrexate $(50 \mathrm{mg}$ per metres squared) given intramuscularly at 6 weeks gestation.

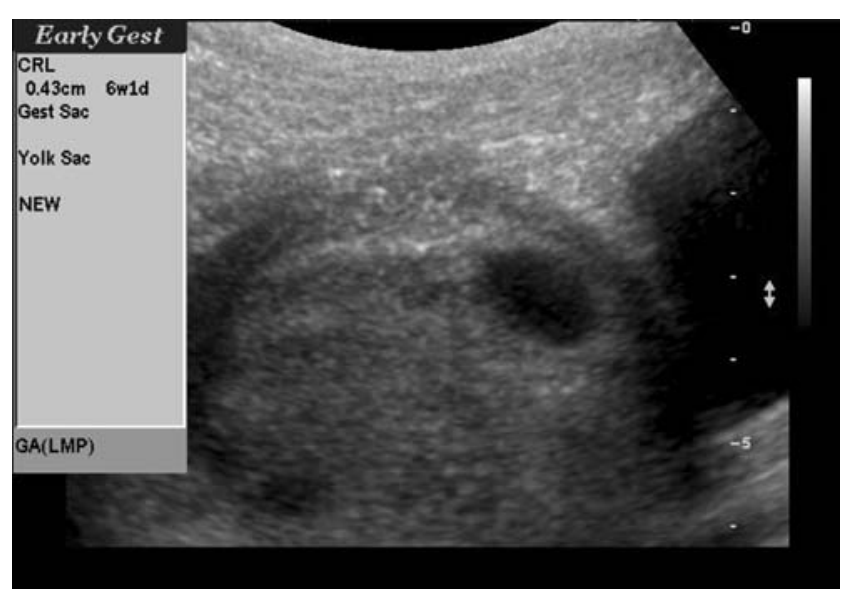

Six days later, the patient reported worsening pain and her BhCG level had risen to $25,300 \mathrm{mIU} / \mathrm{mL}$, with persistent foetal cardiac activity. Secondary to increasing pain, despite her stable status and appropriate counselling, the patient declined further medical treatment and strongly desired surgical intervention. A well-informed management approach amongst the patient, her husband and the departmental team took place. The plan was to pursue an ultrasound-guided dilatation and curettage, backed up by possible intra-amniotic Methotrexate injection or transabdominal embryo aspiration. After cervical dilatation, suction evacuation was performed under transabdominal ultrasound guidance, followed by sharp curettage. The patient's vital signs were closely watched and ultrasound was continued after the procedure to monitor intraabdominal bleeding. Uterotonic agents were not required. The patient's post-operative course was uneventful and her BhCG level declined by $>15 \%$ post-D\&C $(17,400 \mathrm{mIU} / \mathrm{mL})$. The final pathology revealed products of conception, including numerous immature villi, gestational endometrium and decidual reaction with focal necrosis.

\section{Discussion}

We report a case of ectopic pregnancy implanted eccentrically in the caesarean scar in a subject with three prior caesarean deliveries. Ectopic pregnancy remains the leading cause of maternal morbidity and mortality during the first trimester. This rare form of ectopic pregnancy in a caesarean scar is even more concerning for life-threatening complications secondary to the difficulty in diagnosis and lack of optimal treatment.

It has been proposed that caesarean scar pregnancy is brought about by endometrial and myometrial disruption and scarring subsequent to caesarean delivery, leading to implantation in the uterine scar via a microscopic dehiscent tract [1-3]. Predisposing factors for pregnancy in a caesarean scar include multiple prior caesarean deliveries and other uterine surgical procedures, such as curettage, myomectomy, metroplasty or manual removal of the placenta $[4,5]$. These give a risk factor for in-scar implantation because of the increased scar surface area [6].

A pregnancy located in a caesarean scar is considered as high risk because of its early invasion of the myometrium, which may lead to uterine rupture, together with all of its related maternal complications. Hence, prompt and accurate diagnosis is crucial. With the aid of sonographic imaging, criteria for diagnosis have been proposed: (1) empty uterine cavity and cervical canal; (2) development of the gestational sac in the anterior uterine wall of the isthmus (presumed site of the previous lower segment caesarean section scar); (3) evidence of functional trophoblastic circulation on Doppler examination, defined by the presence of an area of increased peritrophoblastic vascularity on colour Doppler examination; and (4) the absence of healthy myometrium between the bladder and sac, allowing differentiation from cervico-isthmic implantation $[4,7]$. 
The caesarean section rate most recently reported by the Centers for Disease Control (CDC) as of 2004 is $29.1 \%$ and will continue to rise with the new trend of "caesarean section on demand" for elective primary section. With the high numbers of caesarean deliveries and the inevitability of subsequent pregnancies, long-term outcomes such as caesarean scar pregnancies must be recognised. Physicians and patients need to be aware of this rare entity as a potential remote consequence of multiple caesarean deliveries, in addition to other known risks, such as placenta previa, accreta and caesarean hysterectomy.

Because of the rarity of this condition, there are only a few case reports in the literature with a discussion of various management options. Non-surgical treatment includes Methotrexate (systemic, local or combined, single versus multiple doses), uterine artery embolisation, potassium chloride and hyperosmolar glucose. Surgical management may be intra-amniotic Methotrexate, surgical excision (via laparotomy or laparoscopy, with possible repair of defect) and dilatation and curettage $[2,7,8]$.

In selecting the mode of treatment, important considerations include the patient's clinical status, size and age of gestational mass, whether to leave the uterine scar defect that may potentially affect future pregnancies, operative risk in case of the need for emergency hysterectomy etc. [9]. In our case, the patient was treated successfully with systemic Methotrexate and curettage. Methotrexate prior to surgery may have downgraded trophoblastic proliferation and decreased to a minimum the risk of bleeding and uterine rupture. In this setting though, the dose of Methotrexate may have been inadequate. This kind of pregnancy may be more vascular and invasive and, therefore, requires higher dosages of Methotrexate. This theory, though, may benefit further exploration.
In conclusion, we report a case of caesarean scar pregnancy that was managed by a combination of medical and surgical management options. Reports such as this will increase obstetricians' awareness of this potentially life-threatening condition in a subject with prior multiple uterine surgeries and facilitate early diagnosis and proper management. We encourage all caesarean scar pregnancies to be reported in order for an optimal approach to be identified.

\section{References}

1. Molinaro TA, Barnhart KT (2007) Ectopic pregnancies in unusual locations. Semin Reprod Med 25:123-130

2. Rotas MA, Haberman S, Levgur M (2006) Cesarean scar ectopic pregnancies: etiology, diagnosis, and management. Obstet Gynecol 107:1373-1381

3. Sugawara J, Senoo M, Chisaka H, Yaegashi N, Okamura K (2005) Successful conservative treatment of a cesarean scar pregnancy with uterine artery embolization. Tohoku J Exp Med 206:261-265

4. Fylstra DL (2002) Ectopic pregnancy within a cesarean scar: a review. Obstet Gynecol Surv 57:537-543

5. Fylstra DL, Pound-Chang T, Miller MG, Cooper A, Miller KM (2002) Ectopic pregnancy within a cesarean delivery scar: a case report. Am J Obstet Gynecol 187(2):302-304

6. Maymon R, Halperin R, Mendlovic S, Schneider D, Herman A (2004) Ectopic pregnancies in a Caesarean scar: review of the medical approach to an iatrogenic complication. Hum Reprod Update 10(6):515-523

7. Yan CM (2007) A report of four cases of caesarean scar pregnancy in a period of 12 months. Hong Kong Med J 13(2):141-143

8. Maymon R, Halperin R, Mendlovic S, Schneider D, Vaknin Z, Herman A, Pansky M (2004) Ectopic pregnancies in caesarean section scars: the 8 year experience of one medical centre. Hum Reprod 19:278-284

9. Chou MM, Hwang JI, Tseng JJ, Huang YF, Ho ES (2004) Cesarean scar pregnancy: quantitative assessment of uterine neovascularization with 3-dimensional color power Doppler imaging and successful treatment with uterine artery embolization. Am J Obstet Gynecol 190(3):866-868 